\title{
An Approach to Accuracy Improvements in the Flexible Machining of Curved Profiles
}

\author{
G. Lanza ${ }^{1, a}$, J. Fleischer ${ }^{1, b}$ and D. Ruch ${ }^{1, c}$ \\ ${ }^{1}$ wbk Institute of Production Science, Universität Karlsruhe (TH) \\ Kaiserstraße 12, 76128 Karlsruhe, Germany \\ a lanza@wbk.uka.de, ${ }^{b}$ fleischer@wbk.uka.de, 'ruch@wbk.uka.de
}

Keywords: Research, clamping, flexible production systems, quality control

\begin{abstract}
This article describes a clamping concept for the flexible machining of spatially curved profiles developed at the wbk Institute of Production Science of the Universität Karlsruhe (TH). Simple geometrical considerations form the basis of the prototypically implemented clamping system design. The approach presented in this article allows for accuracy improvements regarding the positioning of the profile in the clamping system on the basis of markings applied onto the surface of the profile. Besides, the preliminary test rig set up and first results on the detection of the markings by means of digital image processing are presented.
\end{abstract}

\section{Introduction}

Automated small-batch production calls for a high degree of flexibility and adaptability in terms of both equipment and processes. This demand is one of the most urgent requirements and at the same time one of the most difficult to comply with for equipment and processes that are directly related to the products to be manufactured [1]. As far as the Collaborative Research Center (SFB) Transregio 10, which focuses on the small-to-medium batch production of space frame structures, is concerned, the call for a high degree of flexibility poses a major challenge to the handling and machining processes and to in-line quality measurements. Spatially curved profiles require machining along the entire profile length at different positions throughout the whole machining process. Control of the profile geometry has to be performed by a quality assurance system integrated into the machining process.

The highly flexible clamping devices partly used in the aviation industry for small-batch production can variably adapt to a three-dimensional profile contour. The profiles are secured at several flexibly adaptable clamping points along the entire profile length [2]. It must be pointed out, though, that highly flexible fixtures like these are very complex in terms of construction and design, requiring enormous investment. Adapted clamping fixtures, which clamp the profile at several fixed positions, are an alternative to the complex clamping system described before. Here, the clamping elements consist of modular standard clamping elements, which can be swapped manually in order to adapt the fixture to the product $[3,4,5,6]$. Machine concepts for the machining of straight profiles with constant cross-sections allow for the use of geometrically simple clamping system constructions, due to the fact, that the orientation of the profiles surface does not change in reference to the clamping system. If required, the flexibility of these systems can be improved with clamping jaws adapted to the profiles geometry, which also are used for complex cross-sections or thin-walled profiles [7]. The highly flexible clamping systems as well as the adapted clamping fixtures necessitate the feed range of the machines kinematic mechanism to be at least as large as the component itself. For the automated handling of components predominantly additional devices, e.g. industrial robots or special-purpose machines are used. The processes of loading and unloading, however, are manual in small-batch production. In small-batch production, the profile geometry is currently mostly controlled on the basis of individual testing using either coordinate measurement systems or product-specific gauges. Any in-line quality assurance guaranteeing $100 \%$ of control would require enormous time and effort. Taking the above considerations into account, the 
processes for quality assurance regarding profile handling and clamping currently used in the industrial environment are only conditionally suited for automated small-batch production.

For the purpose of eliminating the disadvantages of conventional machine concepts, a novel concept minimizing the above-mentioned downsides of conventional machine technology has been elaborated out of the close collaboration between the two subprojects "Flexible and intelligent gripping systems" and "Combined kinematic handling and machining mechanism" [8]. Figure 1 shows the model of the elaborated machine concept. The profile in question is gripped by an industrial robot, subsequently guided through a flexible clamping system and positioned there. Once the profile has been clamped, it is machined by the kinematic machining mechanism. The profile is repositioned by the industrial robot to allow it to be machined along its entire length. The machining process itself and its features are explained in the following paper of this issue (Accuracy Improvement of a Machine Kinematics for the Product Flexible Machining of Curved Extrusion Profiles).

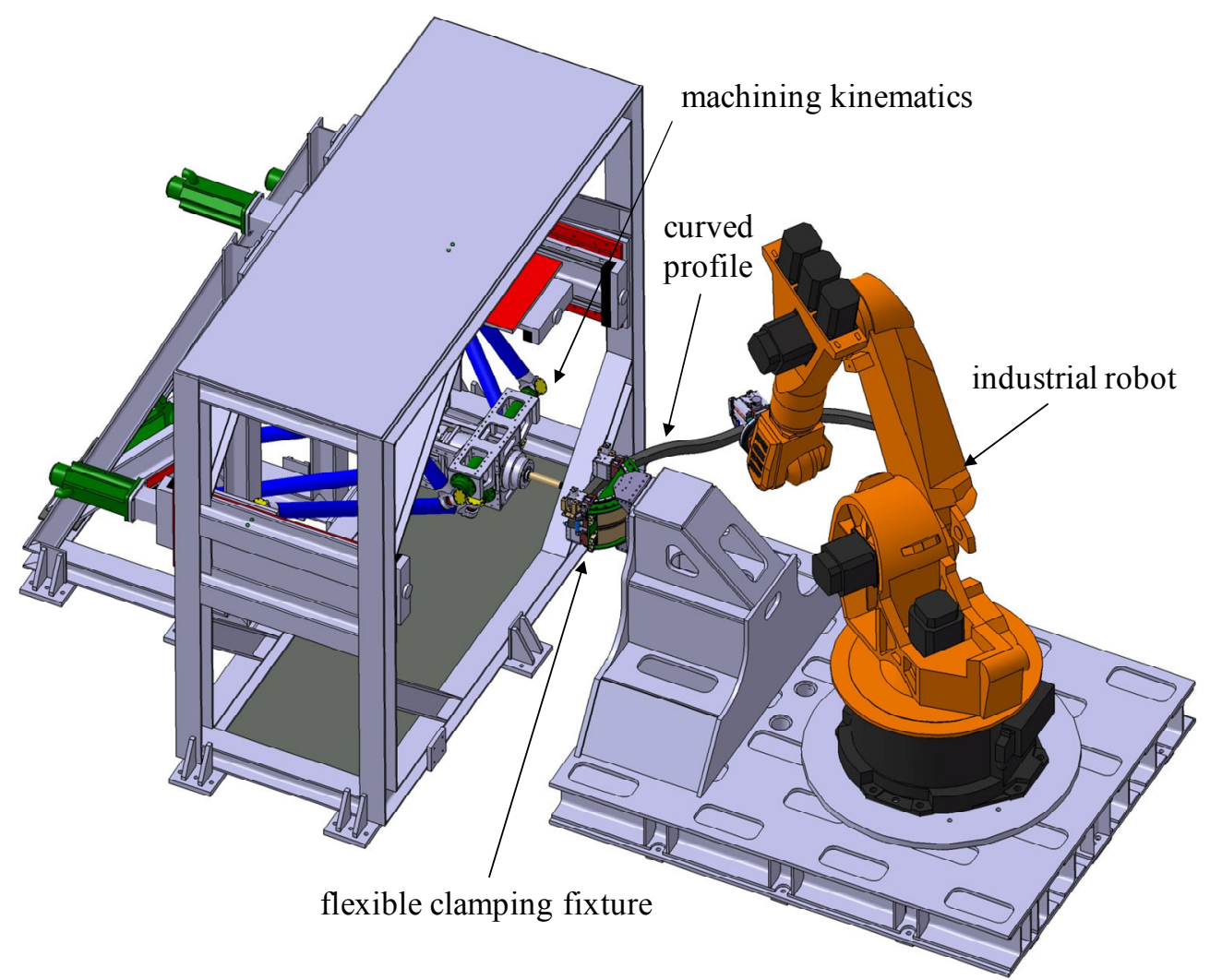

Figure 1: Model of an overall machine concept for the flexible machining of spatially curved profiles

\section{Design of the machining and clamping concept}

In order to decouple the clamping process from a profile's three-dimensional geometrical shape, the clamping concept that has been developed distinguishes between the two functions "clamp profile" and "determine position of profile". If the physical profile contour is reduced to the profile's center line, it will always be possible to position this center line to meet two fixed points P1 and P2 (Figure 2, left). This purely geometrical approach can be used to determine four degrees of freedom of the curve, i.e. two rotational and two translational ones. The two degrees of freedom that are yet to be determined would consist in a movement of the profile along the $\mathrm{x}$ axis and its rotation about it in the local coordinate system, the $\mathrm{x}$ axis being the connecting line between points P1 and P2. If applying this approach to a physical clamping system, it would be possible for almost each profile geometry to meet two support points at the same time. As a physical profile cannot be reduced to its own center line, the number of imaginary support points needs to be raised to four. 
Technology-wise, this approach may take the form of two lines each perpendicular to one another and molded as stop angles (Figure 2, right). Depending on each profile's cross-section, the contact patch between the profile's surface and the stop angle may take the shape of either points or lines. If the profile's cross-section has got a circular shape, there will be one punctiform contact patch between the profile and each of the four stop angles (Figure 2). This serves to determine both, the position of the profile and the above-mentioned profile center line in four degrees of freedom.
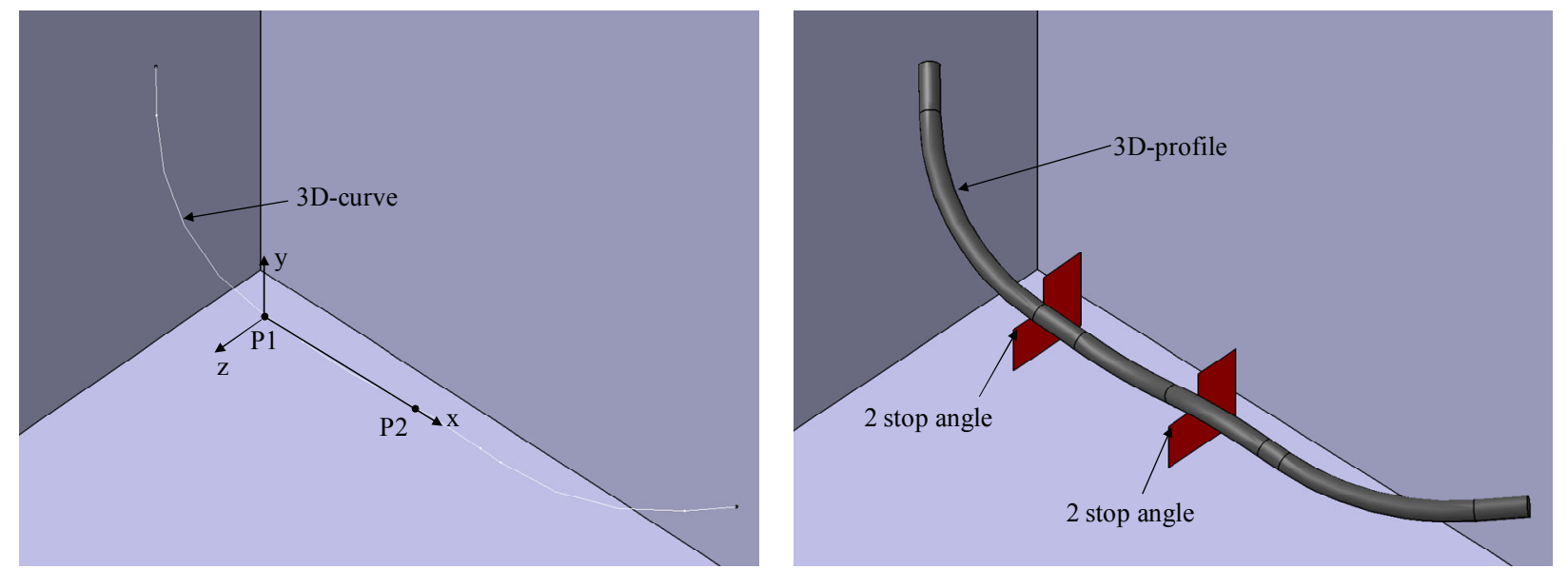

Figure 2: Spatial positioning of a three-dimensional curve

The two degrees of freedom being yet undetermined are narrowed down on the basis of the developing overdetermination of the system by means of a second gripper which is attached to an industrial robot. The function "clamp profile" was implemented by means of two clamping units that are adaptable to any workpiece surface geometry and attached next to the stop angles. This principle is described in detail in [9] and [10]. Machining of the profile takes place between the two clamping units. In case the profile requires machining outside this area, the industrial robot pushes it to the desired location with the gripper, where it is clamped again and machined. The pushing movement requires the stop angles of the prototype to be shaped as stop rollers. This way, the profile surface cannot be damaged during the pushing process. The prototypical design of this machine concept can be seen from Figure 3. The position of the stop rollers has been highlighted with white in the enlarged view of the clamping system shown in Figure 3. This picture does not include the kinematic machining mechanism arranged opposite the clamping system. The gripper has been gimbal-mounted to the chassis with a rotary and a swivel axis in order to ensure that boreholes will always be drilled perpendicular to the profile, for example. Each of these axes has been endowed with an angle measurement system, but neither has got its own drive. Both axes are indirectly driven by the industrial robot which is linked to the clamping system via the gripped profile. The results of the angle measurements are transferred to the robot control for angle readjustment. During machining both axis are mechanical fixed. This enables a direct force flow from the cutting tool over the profile to the machine base [9]. 


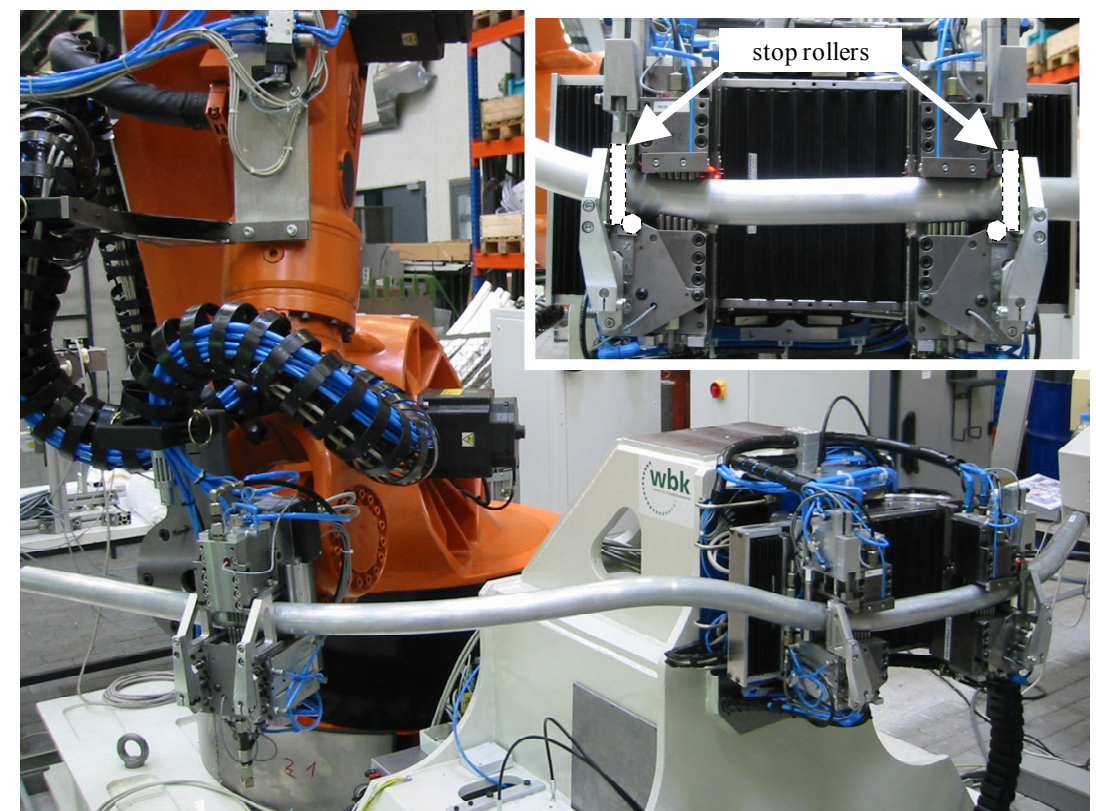

Figure 3: Prototype of the clamping system with clamped profile

\section{An approach to profile positioning}

The two degrees of freedom that cannot be determined on the basis of the mechanical stops have to be identified by means of a metrological approach. The measurement results can either be transferred to the industrial robot for fine positioning of the profile, or to the NC control of the kinematic machining mechanism for a correcting motion of the tool. Therefore, the section is measured from a reference point on the profile surface. The first test results have already been described in [11]. Markings applied onto the profile surface produced the best results in previous tests [12]. If several markings can be geometrically related to each other, these markings can be considered a form of "component-specific gauge" positioned on an imaginary line on the profile surface.

If the gaps between the markings can be made sufficiently accurate to allow for the exact positioning of the profile, the gauge can be considered precise in relation to a reference mark. The gauge can then be used as the measurement standard.

The following paragraph describes a way to identify the position of the profile by the example of a linear profile with a circular cross-section under these circumstances. The industrial robot positions the profile at the initial target position where the reference mark has been made. If there is no absolute position allocation for the markings, they need to be approached by the industrial robot one by one and analogically recorded in an incremental measurement system. The gaps then need to be totaled. Another possibility would be to give the marking an absolute reference by adding additional information. The simplest variant would be a number combination or a different form of coding. It might also be possible to integrate the absolute reference into the shape of the marking, for instance through circles with different diameters. The center of the circle would refer to the position, whereas the surface area would relate to the absolute reference. Other possible shapes are looked at in the following work steps.

In case it is impossible to achieve sufficient accuracy for the markings on the profile, there are two options. The markings can either be put on with repetitive accuracy or with variable accuracy, i.e. "subject to errors". Either way, it would be impossible to simply read out the markings at a random point on the profile. This being the case, the gauge on the profile could not be used as the measurement standard. It would be possible, though, to use a measurement system like a image processing system, for example, to measure the gap between two adjoining markings $M_{i}$ and $M_{i+1}$. The measurement would have to be carried out on the basis of a reference mark as well. If a gap 
between two markings is too large to be measured with sufficient accuracy by one measurement system only, the distance between the markings

$$
A_{M_{i} \rightarrow M_{i+1}}=a+\Delta a
$$

needs to be determined via a second measurement system positioned at the known distance a to the first measurement system (Figure 4). $\Delta \mathrm{a}$ is the deviation between the target and the actual position of the marking $M_{i+1}$. The resulting actual distance $A_{M_{i} \rightarrow M_{i+1}}$ between two adjoining markings needs to be buffered in a calibration file and allow for allocation to the respective marking at any point in time along the process chain. To permit calibration of the gauge along the entire profile length, the profile needs to be moved forward to the next marking by the industrial robot, a process that requires repetition until all markings have been recorded and stored in the calibration file.

For this approach to be applied to curved profiles, the profile geometry between the adjoining markings needs to be known also, because the above-described procedure is limited to the identification of the projected gap between two markings (Figure 4). Given that the geometry between two markings is known, the developed length of the imaginary surface line can be calculated. Figure 4 shows the schematic illustration of the metrological design with two flat profiles, one linear and one with a constant radius.

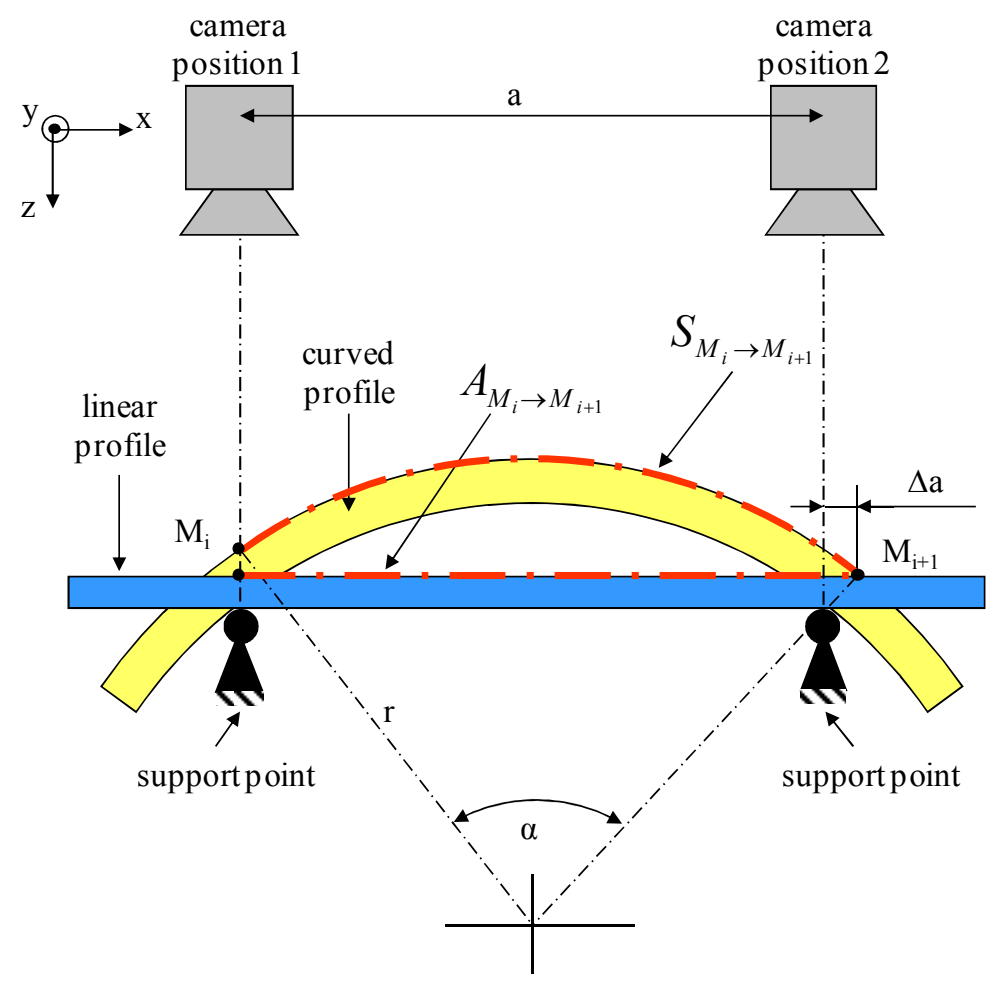

Figure 4: Schematic illustration of the calibration of two adjoining markings

In both cases, the fault $\Delta \mathrm{a}$ is measured on the basis of image processing, and then used to determine the actual gap between the two adjoining markings $M_{i}$ and $M_{i+1}$. The developed length of the curved profile exceeds the gap between the markings on the linear profile as $S_{M_{i} \rightarrow M_{i+1}}$. The length $S_{M_{i} \rightarrow M_{i+1}}$ can be calculated with 


$$
S_{M_{i} \rightarrow M_{i+1}}=\frac{\pi r}{180^{\circ}}\left[\arcsin \left(\frac{a}{2 r}\right)+\arcsin \left(\frac{\left(\frac{a}{2}+\Delta a\right)}{r}\right)\right]
$$

for a segment of a circle with the known outside radius $r$ and the measured fault $\Delta \mathrm{a}$. In order to move the profile to other positions, it needs to be continuously forwarded from one marking to the next, and the measurement process needs to be repeated as many times as necessary until the desired position has been reached. The markings should be applied onto the profile with a gap as big as possible between them. This prevents unnecessarily slowing down the process and minimizes the measurement faults resulting from totaling as much as possible.

If the profile geometry between two markings is not known, it needs to be metrologically identified, too. If the described procedure is applied to curved profiles with a non-constant radius or to spatially curved profiles, the mathematical description of the profile contour requires a splin interpolation. An optical sensor measuring two dimensions and operating on the basis of the triangulation method is used to identify the profile contour between the support points. The profile sensor is positioned opposite the profile and moved across it by means of a linear axis. A schematic illustration of the design can be seen from Figure 5. The distance $\Delta z\left(x_{i}\right)$ between the sensor and the profile is measured at different points $x_{i}$. If the profile is spatially curved, the transverse direction $\Delta y\left(x_{i}\right)$ of the profile sensor is additionally measured at each measuring point $x_{i}$. The resulting measurement results can be used to calculate the length of an imaginary surface line between two adjoining markings, approximated via a cubic spline, by means of a line integral. The calculated length matches the gap between the two markings as stored in the calibration file.

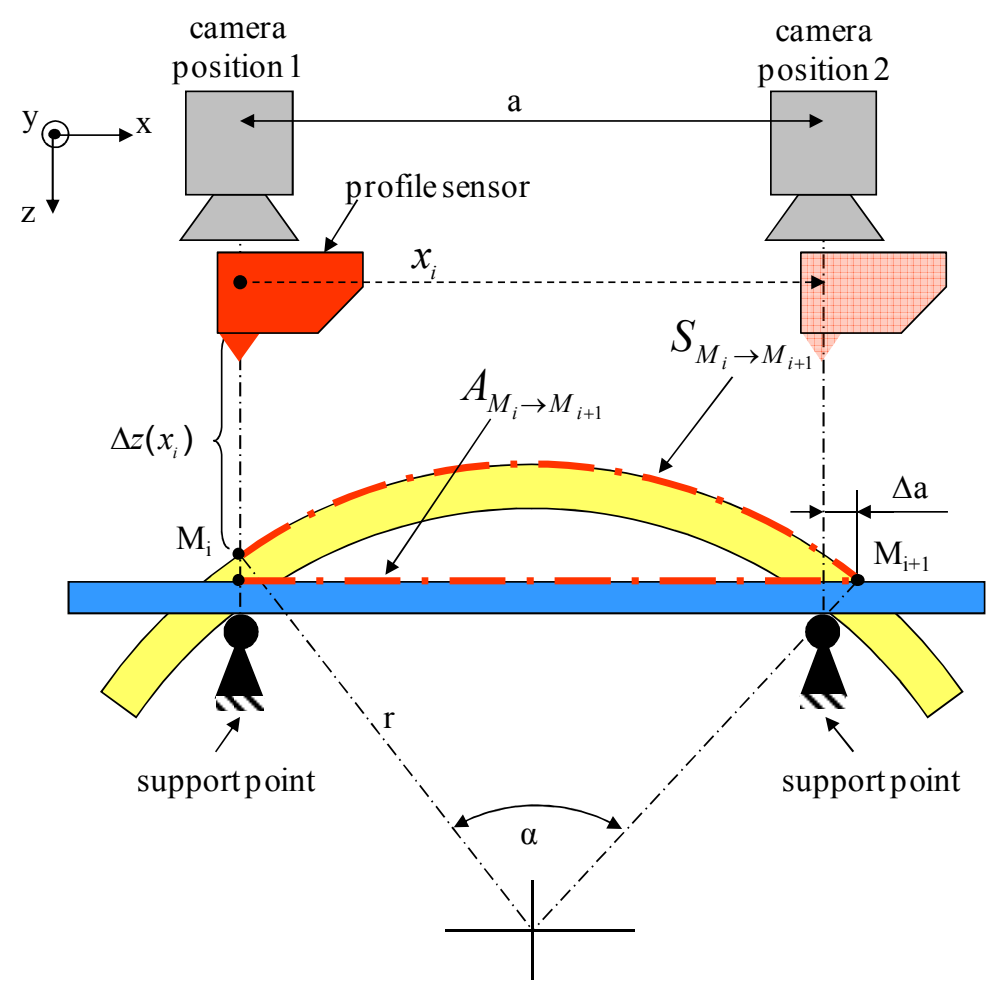

Figure 5: Design of the measurement devices for profile positioning and contour measurement

For this design to be implemented and for the described measurements to be performed in-line, the described measurement system needs to be integrated into the kinematic machining mechanism opposite the clamping system in the long run. It will then be used to achieve the correct positional 
arrangement of the measuring devices in relation to the profile. To allow for the profile to be measured according to the above-described approach and for the gauge to be read, it needs to be ensured that the gripping system is correctly aligned during the measurements, i.e. that the rotatory and the swivel axes of the clamping system are each set to $0^{\circ}$ by the industrial robot. Any angle other than $0^{\circ}$ would cause the mechanical guide rollers to tip in relation to the measurement system and thus, leads to faulty camera and profile sensor measurements. Figure 6 shows the relevant principle of the control process of the industrial robot and the clamping system for a gauge that is "subject to error".

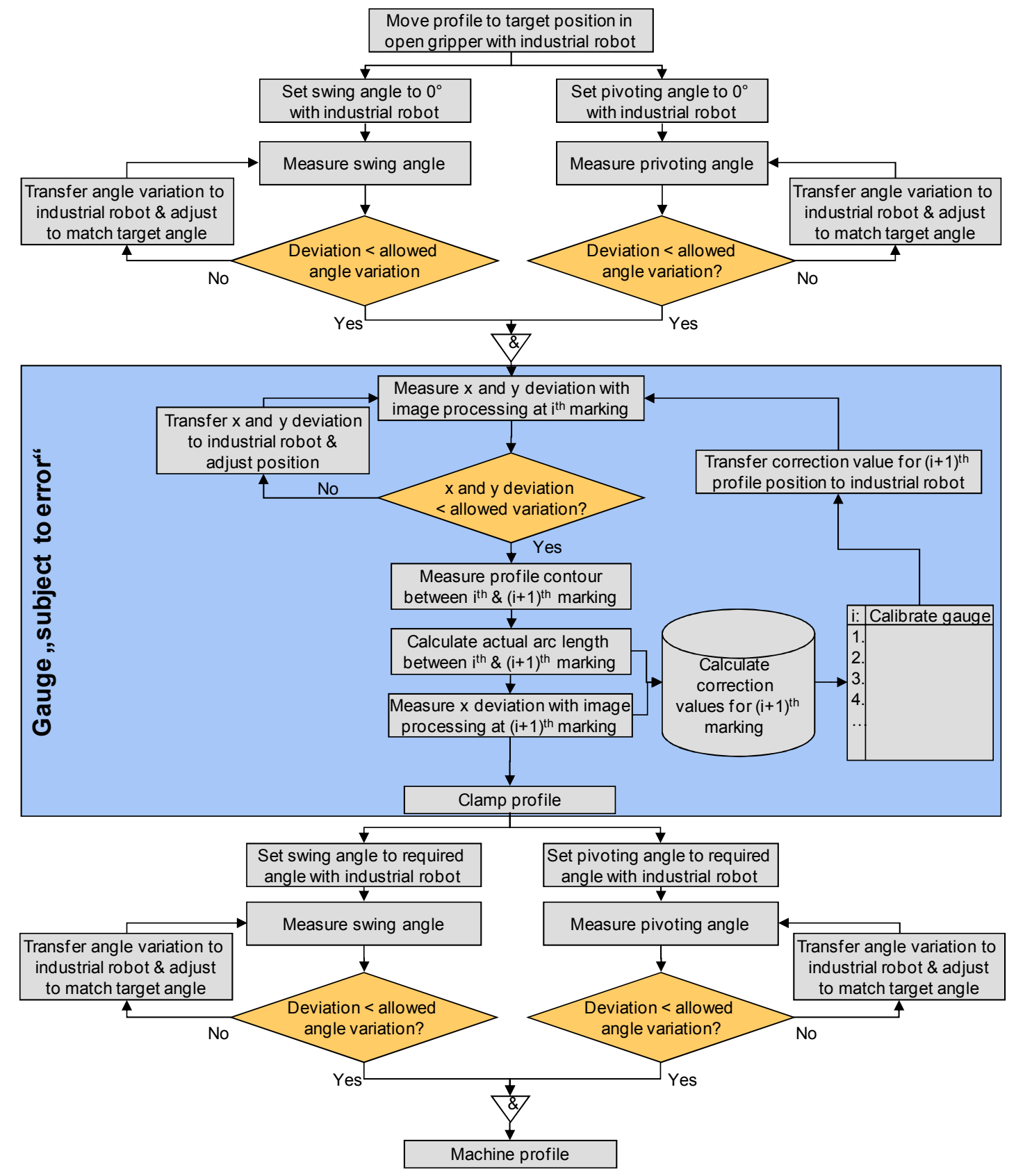

Figure 6: Control process during profile positioning

\section{Marking application and detection}

Numerous tests showed that the best and easiest way to position and align the profile is by using markings on the surface of the profile. The tested markings were applied onto the profiles using a marking laser. This method offers the advantage of being able to apply the markings with high accuracy and speed. These are the prerequisites to be met if the markings are to be applied simultaneously with the extrusion process, i.e. in-line, to avoid an additional process step having to 
be integrated into the process chain. In order to prevent the marking process from interspersing the surface of the profiles with notches, the labeling process is adjusted to allow for a low application of energy into the material only to just slightly roughen the surface. The resulting markings are not easily visible, though, since depending on the incidence of light contrast might be low. This poses a challenge to an automated reading of the markings by means of a camera system. For this reason, tests with different lighting equipment were carried out. The highly reflecting aluminium surface may have a negative impact on image evaluation. The reflections may be reduced by using diffuse or polarizing light, for example [13]. If the profile surface is illuminated with polarizing light, the light reflected from the profile surface will also be polarized. This very light can be blocked by means of an analyzer installed in front of the camera objective. The analyzer is only lighttransmissive if the light does not vibrate at the same polarization level as the reflected light. Faults on the profile surface or the surface sections roughened by the laser beam scatter the light diffusely, thus allowing a large part of this light to pass through the analyzer. As a consequence, the image produced by the camera shows the faint markings of the profile as bright against the unlabeled profile surface creating a high-contrast image, thus facilitating digital image processing (Figure 7, below).

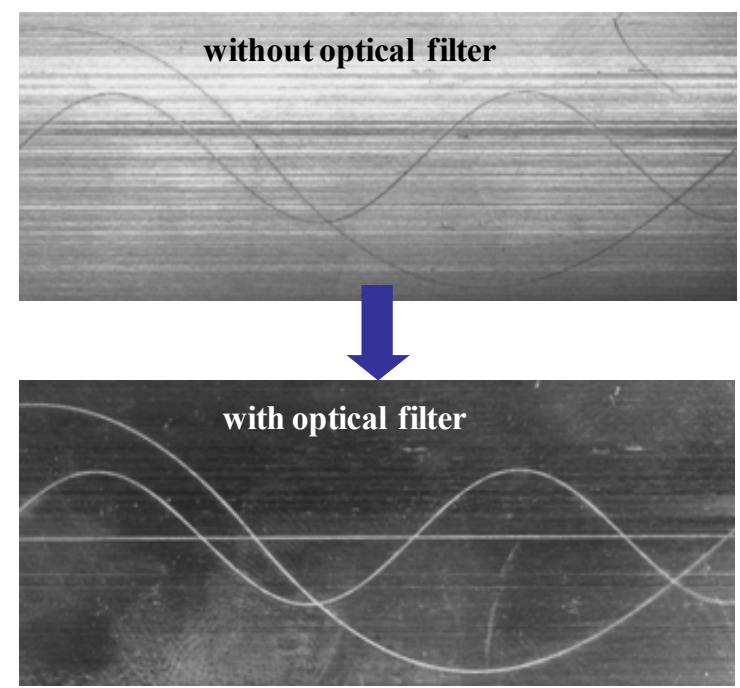

Figure 7: With and without optical filter

In order to measure the position deviation of a profile along its longitudinal axis and its rotation about it via image processing, a program was set up which is able to calculate the coordinates of the intersecting point of a marked cross and to transfer them to a primary control like the robot control, for example, via a serial interface (Figure 8, left). The absolute allocation of this intersecting point is ensured by a number combination positioned alongside the cross which is identified via character recognition. Figure 8, right, shows the binarized image of the marking.
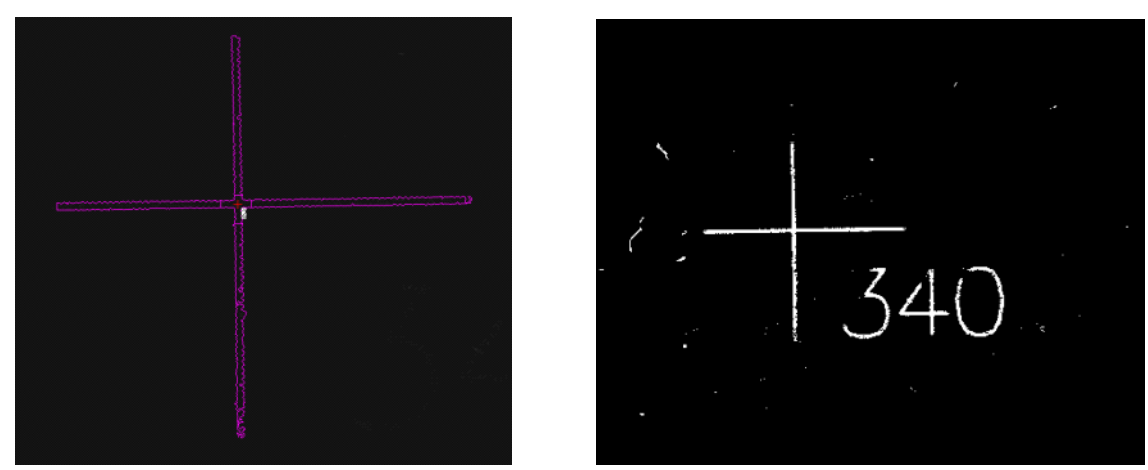

Figure 8: Illustration of cross and number 


\section{Set-up of the preliminary test rig and first results}

Prior to integrating the measuring devices schematically illustrated in Figure 4 and Figure 5 (camera $1 \& 2$, profile sensor and linear axis with measurement system) into the gripping system or the kinematic machining mechanism respectively, they were incorporated into the preliminary test rig in a first step (Figure 9). The test rig serves to test the approach to profile positioning by means of component-specific markings and to profile measurements under ideal conditions and to identify the achievable accuracies. The theoretical resolution of the camera resulting from this test setup totals approx. $17,5 \mu \mathrm{m}$ for a display detail of approx. $28 \times 25 \mathrm{~mm}$.
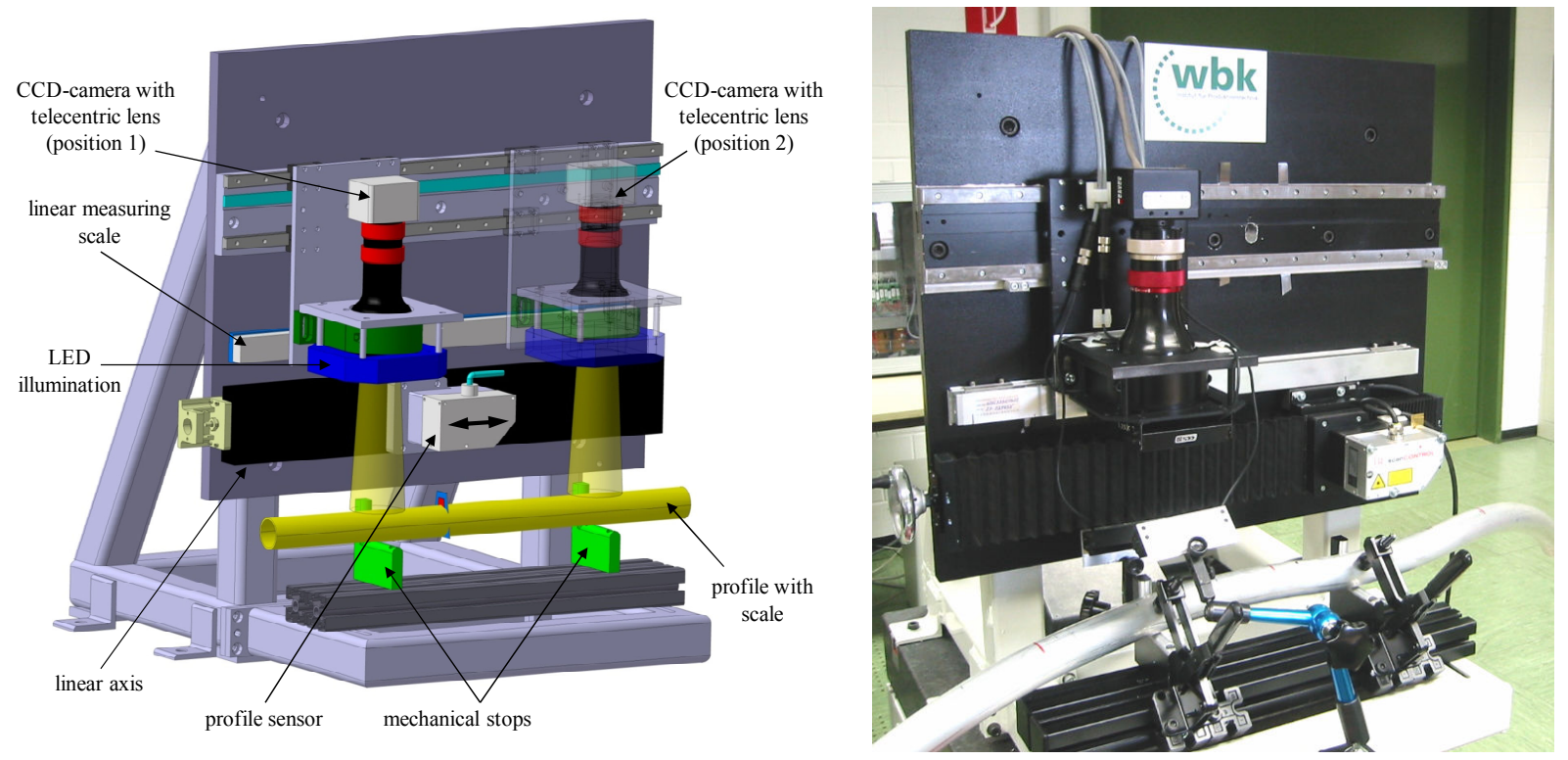

Figure 9: Preliminary test rig for profile measurements

In order to evaluate the system accuracy - camera with objective and lighting - one marking was measured several times without moving the profile between measurements. The $\mathrm{x}$ and $\mathrm{y}$ positions were determined in 400 measurements. Figure 10 shows the measured deviations. It became clear that the $\mathrm{x}$ position can be determined with a standard deviation of $\sigma_{x}=1,8 \mu \mathrm{m}$, whereas this value amounts to $\sigma_{y}=3,0 \mu \mathrm{m}$ for $\mathrm{y}$. The slightly lower accuracy in y direction partly originates from the fact that the profile surface was strongly curved $(\mathrm{r}=20 \mathrm{~mm})$. The conversion of the y deviation into the profile twist results in a standard deviation of $\sigma=31,2$ " for a profile with a radius of $20 \mathrm{~mm}$. These results suggest that the use of an image processing system for the detection of markings is not only flexible but also allows for a high degree of accuracy. 


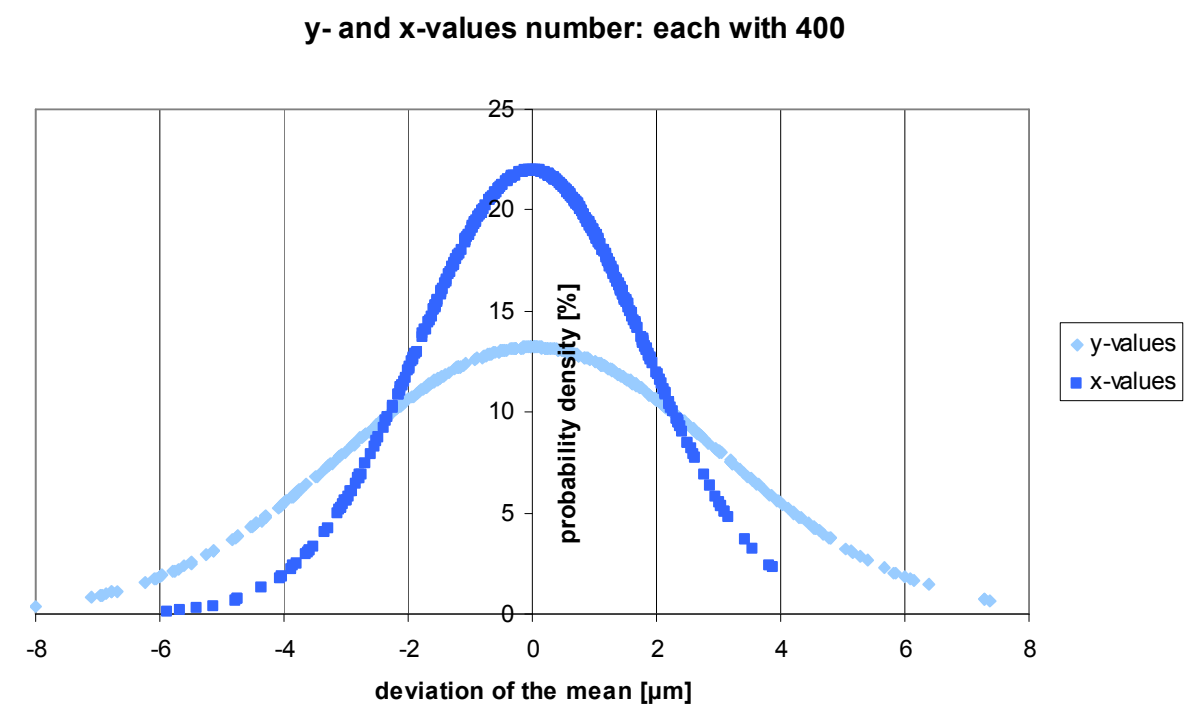

Figure 10: Probability density above the $\mathrm{x}$ and $\mathrm{y}$ deviations during marking detection

\section{Summary and outlook}

This article presents an approach that allows for the flexible clamping and machining of spatially curved profiles by means of a novel machine concept elaborated at the wbk Institute of Production Science. It then moves on to explain, on the basis of the previous results, the use of mechanical stops to determine the position and alignment of almost any profile contour inside the prototypically implemented clamping system. An approach based on profile-specific markings was presented allowing for metrological identification of the non-identifiable degrees of freedom via mechanical stops. The respective accuracy achievable when applying the above-mentioned gauge within the process chain was identified. Depending on the accuracy of the gauge, different metrological and control methods need to be chosen for the determination of position and alignment. In addition, the first results from gauge detection on the basis of digital image processing were presented.

Subsequent research will look into the ideal design of the markings on the profile surface and the achievable positioning accuracies with physical clamping systems on the basis of the abovedescribed approach. Besides, the approach to profile positioning with gaugess subject to errors will be extended in that the entire three-dimensional profile contour is to be identified in-line on the basis of the measurement results gained from the profile sensor.

\section{Acknowledgment}

This paper is based on investigations of the Collaborative Research Center SFB/TR10 which is kindly supported by the German Research Foundation (DFG).

\section{References}

[1] A. Trummer, H. Wiebach: Vorrichtungen der Produktionstechnik. Vieweg-Verlag (1994)

[2] Homepage Fooke GmbH, http://www.fooke.de

[3] Fleischer, J.; Denkena, B.; Winfough, B.; Mori, M.: Workpiece and Tool Handling in Metal Cutting Machines. in: Annals of the CIRP, Vol. 55/2 (2006), pp. 817-839

[4] AMF Produkt Katalog, www.amf.de, (08.2007)

[5] Horst Witte Gerätebau: Hauptkatalog Alufix, Sicherheit mit System, (2007)

[6] Hesse, S.; Krahn, H.; Eh, H.: Betriebsmittel Vorrichtung, Kommentierte Ausführungsbeispiele, Hanser Fachbuchverlag, (2002) 
[7] Homepage ARLA Maschinentechnik GmbH, http://www.arla.de

[8] Fleischer, J.; Schmidt-Ewig, J.P.: Innovative Machine Kinematics for Combined Handling and Machining of Three-Dimensional Curved Lightweight Extrusion Structures. in: Annals of the CIRP, Vol. 54 (2005), pp. 317-320

[9] Fleischer, J.; Lanza, G.; Ruch, D.: Flexible and intelligent gripping technology for machining and handling of spatially curved extruded aluminum profiles. in: Advanced Materials Research, Vol. 10 (2006), pp.153-162

[10] Fleischer, J.; Ruch, D.: Flexibles Spannen räumlich gekrümmter Profile - Form- und konturflexibles Spannen räumlich gekrümmter Aluminium-Strangpressprofile. in: wtWerkstattstechnik online, Jahrgang 95, Ausgabe 09 (2005), pp. 712-716

[11] Fleischer, J.; Lanza, G.; Ruch, D.: Using Part-Specific Scales for Positioning of Spatially Curved Profiles in a Highly Flexible Clamping System. in: Production Engineering Vol. XIII/2 (2006), pp. 39-42

[12] Lanza, G.; Fleischer, J.; Ruch, D.: Flexible und intelligente Greiftechnik. in: FortschrittBerichte VDI Reihe 2 Nr. 661, VDI-Verlag Düsseldorf (2007), ISBN 9783183661022, pp. 371 $-396$

[13] C. Demant, B. Streicher-Abel, P. Waszkewitz: Industrielle Bildverarbeitung - Wie optische Qualitätskontrolle wirklich funktioniert, 2. Auflage, Springer-Verlag (2002) 